\title{
UNIFICATION AND INTERPRETATION OF FUZZY SET OPERATIONS
}

\author{
S.K. Kashyap \\ Scientist, Flight Mechanics and Control Division \\ National Aerospace Laboratories \\ P.B. No. 1779, Bangalore 560 017, India \\ E-mail:sudesh@yahoo.com
}

\author{
J.R. Raol \\ Head, Flight Mechanics and Control Division \\ National Aerospace Laboratories \\ P.B. No. 1779, Bangalore 560 017, India \\ E-mail:jrraol@yahoo.com
}

\begin{abstract}
Fuzzy Logic (FL) facilitates modeling of within-the-event uncertainty. The crisp logic models the 'between the events' uncertainty. Fuzzy techniques in the form of approximate reasoning aid decision making with powerful reasoning capabilities. The FL techniques have been used in varieties of applications: i) image analysis, detection of edges, feature extraction, classification, and clustering, ii) parameter estimation of unknown dynamic systems, iii) home appliances - washing machine, air conditioning systems, and iv) decision fusion, situation and threat assessment. In this paper we study FL concepts, fuzzy sets, several membership functions and their properties, FL operators, fuzzy proposition and attempt to bring out relationships, interconnectivities, contradistinctions between various operations/operators and unification. Illustrative example is presented to make the above concepts very clear.
\end{abstract}

Keywords: Fuzzy operators, Fuzzy inference, decision fusion, situation assessment

\section{Introduction}

Zadeh's Fuzzy Logic[2] facilitates modeling of certain kind of uncertainty. Fuzzy techniques in the form of approximate reasoning provide decision support and expert system with powerful reasoning capabilities. Specifically we study triangular norms, triangular co -norms, fuzzy implication process using s-norm, fuzzy implication methods and provide comparative evaluation with numerical simulation in the context of fuzzy inference system (see Fig. 5).

\section{Triangular-norm (t-norm)}

There are numerous ways to define AND operation in fuzzy logic. In fuzzy terminology we call this as triangular-norm or t-norm. The intersection of two fuzzy sets $A$ and $B$ is specified by binary mapping $T$ (t-norm) on the unit interval; that is, a function of the form

$T:[0,1] X[0,1] \rightarrow[0,1]$ or

more specifically $\mu_{A \cap B}(u)=T\left(\mu_{A}(u), \mu_{B}(u)\right)$

Triangular-norm operators are used in FIS: to combine the clauses in antecedent part of a given rule (e.g. "IF $u_{1}$ is $A_{1}$ AND $u_{2}$ is $A_{2}$ "), and in fuzzy implication process - maps the input fuzzy sets into output fuzzy sets. The different definitions of the t-norms that are frequently used as fuzzy intersections are as follows:

i) Standard intersection: $T(x, y)=\min (x, y)$

ii) Algebraic product: $\quad T(x, y)=x \bullet y \quad$ (Zadeh)

iii) Bounded difference: $\quad T(x, y)=\max (0, x+y-1)$

iv) Drastic intersection: $\quad T(x, y)= \begin{cases}x & \text { when } y=1 \\ y & \text { when } x=1 \\ 0 & \text { otherwise }\end{cases}$

Here, $x=\mu_{A}(u), y=\mu_{B}(u), \quad$ and $\quad u \in U . \quad$ A "fuzzy intersection/t-norm" $T$ is binary operation on the unit interval that satisfies at least the following axioms for all $x, y$ and $z$ in the range $[0,1]$.

Axiom 1: $T(0,0)=0, T(x, 1)=T(1, x)=x$ (boundary condition)

Axiom2: $y \leq z$ implies $T(x, y) \leq T(x, z)$ (monotonicity)

Axiom $3: T(x, y)=T(y, x)$ (commutativity)

Axiom4: $T(x, T(y, z))=T(T(x, y), z) \quad$ (associativity)

Consider the fuzzy sets $A$ and $B$ defined by membership functions 'trimf' \& 'trapmf' (inbuilt MATLAB functions) respectively as:

$$
\begin{aligned}
& \mu_{A}(u)=\operatorname{trimf}(u,[a, b, c]) \\
& \mu_{B}(u)=\operatorname{trapmf}(u,[d, e, f, g])
\end{aligned}
$$

Here, parameters $a, c$ locate the "bases", and $b$ the "peak" of the membership function $\mu_{A}(u)$. Similarly the parameters $d$ and $g$ locate the "bases" of the trapezoid membership function $\mu_{B}(u)$ and $e \& f$ its "shoulders". The typical values of the parameters could be $a=3, b=6, c=8, d=1$, $e=5, f=7$, and $g=8$. The time history of discrete input $u$ to the fuzzy sets $A$ and $B$ is: $u=0,1,2,3,4,5,6,7,8,9,10$. The fuzzified values of the input $u$, passed through membership functions $\mu_{A}(u)=\operatorname{trimf}(u,[a, b, c]) \quad$ (see fig. 1) and $\mu_{B}(u)=\operatorname{trapmf}(u,[d, e, f, g]$ ) (see fig. 2 ), are as follows:

$\mathrm{x}=\mu_{\mathrm{A}}(\mathrm{U})=\{0 / 0+0 / 1+0.0 / 2+0.0 / 3+0.33 / 4+0.667 / 5+1 / 6+0.5 / 7+0 / 8+0 / 9+0 / 10\}$ $y=\mu_{B}(u)=\{0 / 0+0 / 1+0.25 / 2+0.5 / 3+0.75 / 4+1.000 / 5+1 / 6+1.0 / 7+0 / 8+0 / 9+0 / 10\}$

The computed values are pictorially represented in fig. 3 for triangular-norms. 


\section{Triangular - conorm (t-conorm/s-norm)}

The other possible replacement of OR is defined by Zadeh (1965). Like that there are numerous ways to define OR operation in fuzzy logic. In fuzzy terminology we call this as triangular-conorm or s-norm and defined as follows. The union of two fuzzy sets $A$ and $B$, is specified by a binary operation on the unit interval; that is, a function of the form

$$
S:[0,1] X[0,1] \rightarrow[0,1] \text { or }
$$

more specifically $\mu_{A \cup B}(u)=S\left(\mu_{A}(u), \mu_{B}(u)\right)$

This fuzzy operator is used for the following purposes: to combine the clauses in antecedent part of a given rule (e.g. "IF $u_{1}$ is $A_{1}$ OR $u_{2}$ is $A_{2}$ "). The different definitions of the $\mathbf{t}$ conorms that are frequently used as fuzzy unions are as follows:

i) Standard union : $S(x, y)=\max (x, y)$

ii) Algebraic sum : $S(x, y)=x+y-x \bullet y$ (Zadeh)

iii) Bounded sum : $S(x, y)=\min (1, x+y)$

iv) Drastic union $: S(x, y)=\left\{\begin{array}{l}x \text { when } y=0 \\ y \text { when } x=0 \\ 1 \text { otherwise }\end{array}\right.$

where $x=\mu_{A}(u), y=\mu_{B}(u)$, and $u \in U$.

A "fuzzy union/t-conorm/s-norm" $S$ is binary operation on the unit interval that satisfies at least the following axioms for all $x, y$ and $z$ in the range $[0,1]$.

Axiom 1: $S(1,1)=1, S(x, 0)=S(0, x)=x \quad$ (boundary condition)

Axiom2: $y \leq z$ implies $S(x, y) \leq S(x, z) \quad$ (monotonicity)

Axiom3: $S(x, y)=S(y, x) \quad$ (commutativity)

Axiom4: $S(x, S(y, z)=S(S(x, y), z) \quad$ (associativity)

The most important additional requirements for fuzzy union are expressed by the following axioms:

Axiom 5: $S$ is continuous function (continuity)

Axiom 6: $S(x, x)>x \quad$ (superidempotency)

Axiom7:

$x 1<x_{2}$ and $y 1<y 2$ implies $S(x 1, y 1)<S\left(x 2, y_{2}\right)$ (strict monotonicity)

The computed values are pictorially represented in fig. 4 .

\section{Comparison of fuzzy inference method}

In order to compare the various fuzzy implication methods the norms $(\|\|)$ of the so-called relational matrices are computed in Table 1. We see from the column under $\left\|R^{T}\right\|$ that the standard intersection is relatively stronger than the results of other t-norms. Similarly drastic union (under column $\left\|R^{S}\right\|$ ) is relatively stronger than the other s-norms. We also see that the norms under the column $\left\|R^{S}\right\|$ are relatively strong as compared to the norms under column $\left\|R^{T}\right\|$. The most of the norms under column $\left\|R^{T S}\right\|$ fall between the norms under columns $\left\|R^{T}\right\|$ and $\left\|R^{S}\right\|$ because implication methods under it, partially utilize the t-norm and s-norm operators. If we compare the different fuzzy implication rules in terms of their implementation, Zadeh's implication rule is somewhat difficult to implement in any practical application whereas Mamdani and Larsen's implication rules have found extensive application in practical control engineering due to their computational simplicity. Mamdani's implication rule is computationally faster and has been often used in many fuzzy logic based systems.

\section{Conclusions}

Various fuzzy operations are studied and interpretation/unification has been attempted in this paper with some illustration.

\section{References}

[1] Kosko, B., 1997. Fuzzy Engineering. Prentice Hall, Upper Saddle River, New Jersey.

[2] Zadeh, L. A., 1965. Fuzzy sets. Information and Control Jl., Vol. 8, pp. 338-353.

Table 1: Norms of relational matrices

\begin{tabular}{|c|c|c|c|c|c|}
\hline $\begin{array}{c}\text { Implication } \\
\text { methods }\end{array}$ & $\left\|R^{T}\right\|$ & $\begin{array}{c}\text { Implication } \\
\text { methods }\end{array}$ & $\left\|R^{S}\right\|$ & Implication methods & $\left\|R^{T S}\right\|$ \\
\hline $\begin{array}{c}\text { Standard } \\
\text { intersection } \\
\text { Mamdani } \\
\text { implication }\end{array}$ & 1.5553 & Standard union & 2.8686 & $\begin{array}{c}\text { Arithmetic rule of fuzzy } \\
\text { implication - Zadeh / } \\
\text { Lukasiewicz implication }\end{array}$ & 3.3911 \\
\hline Algebraic product & 1.3594 & Algebraic sum & 3.1095 & Max. rule of fuzzy implication & 2.8065 \\
\hline Bounded difference & 1.2356 & Bounded sum & 3.3699 & $\begin{array}{c}\text { Standard sequence fuzzy } \\
\text { implication }\end{array}$ & 2.8766 \\
\hline Drastic intersection & 1.0954 & Drastic union & 4.0000 & Boolean fuzzy implication & 2.8278 \\
\hline & & & & Goguen's fuzzy implication & 3.3102 \\
\hline
\end{tabular}




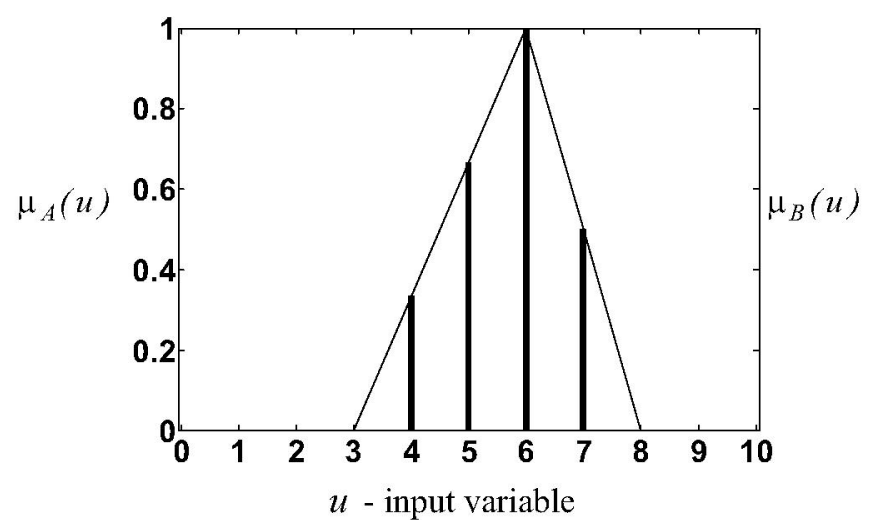

Fig. 1: Triangular shaped membership function

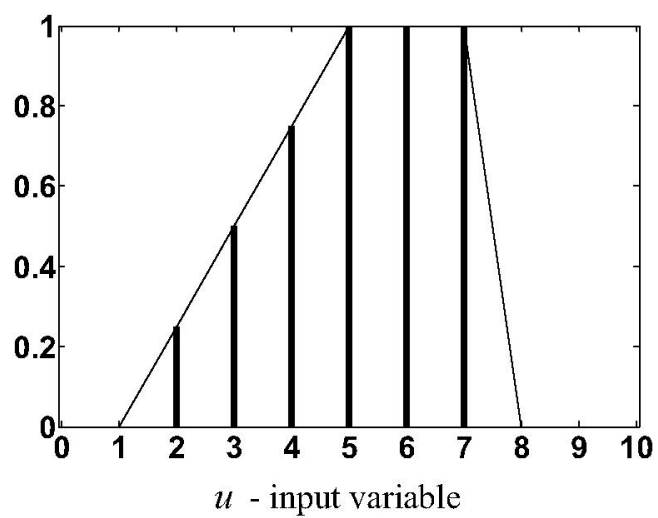

Fig. 2: Trapezoidal shaped membership function

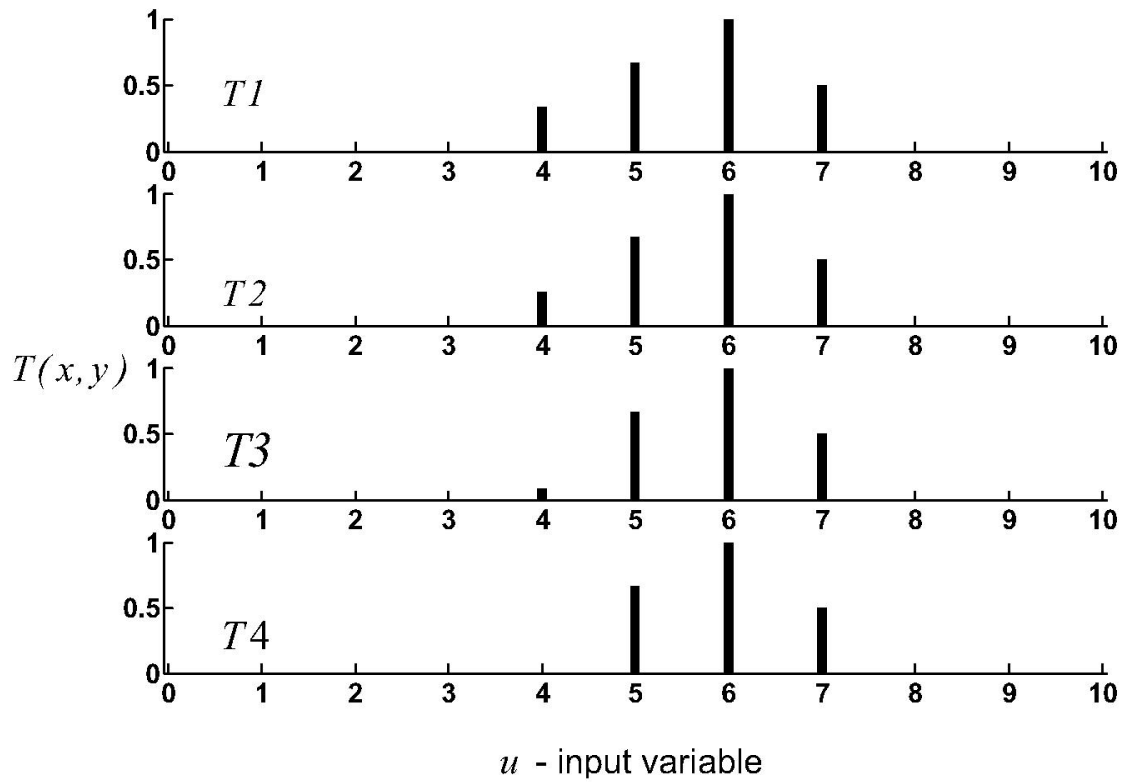

Fig. 3: Pictorial representation of t-norms

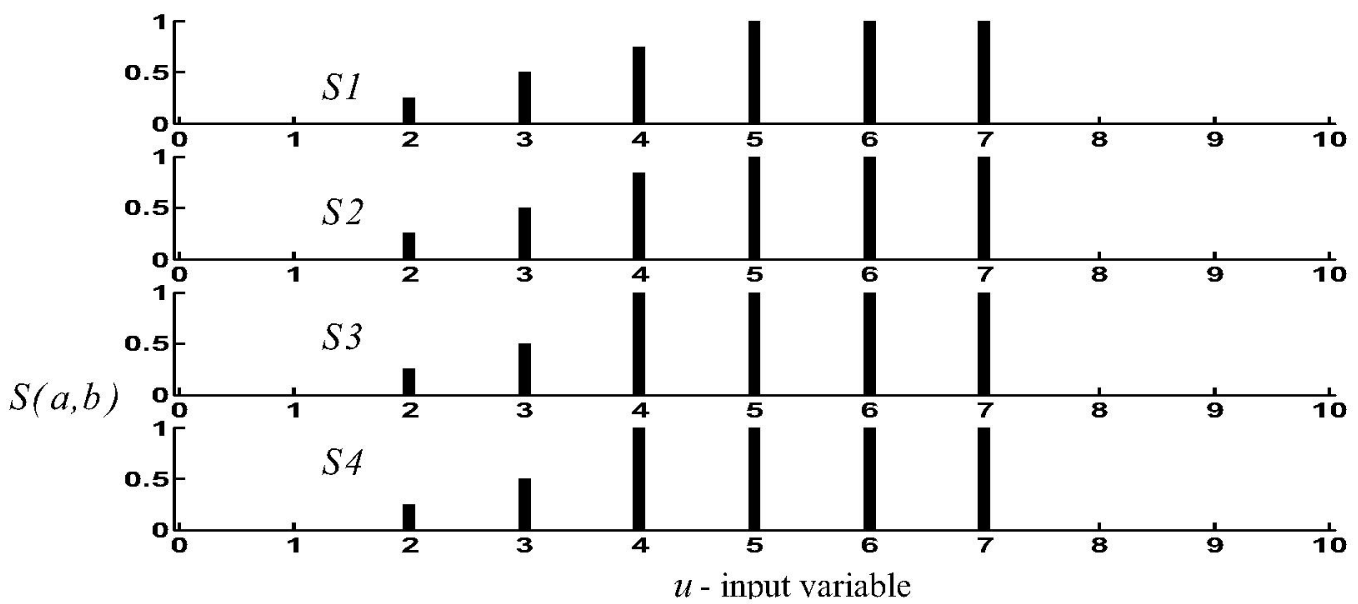

Fig. 4: Pictorial representation of t-conorms or s-norm 


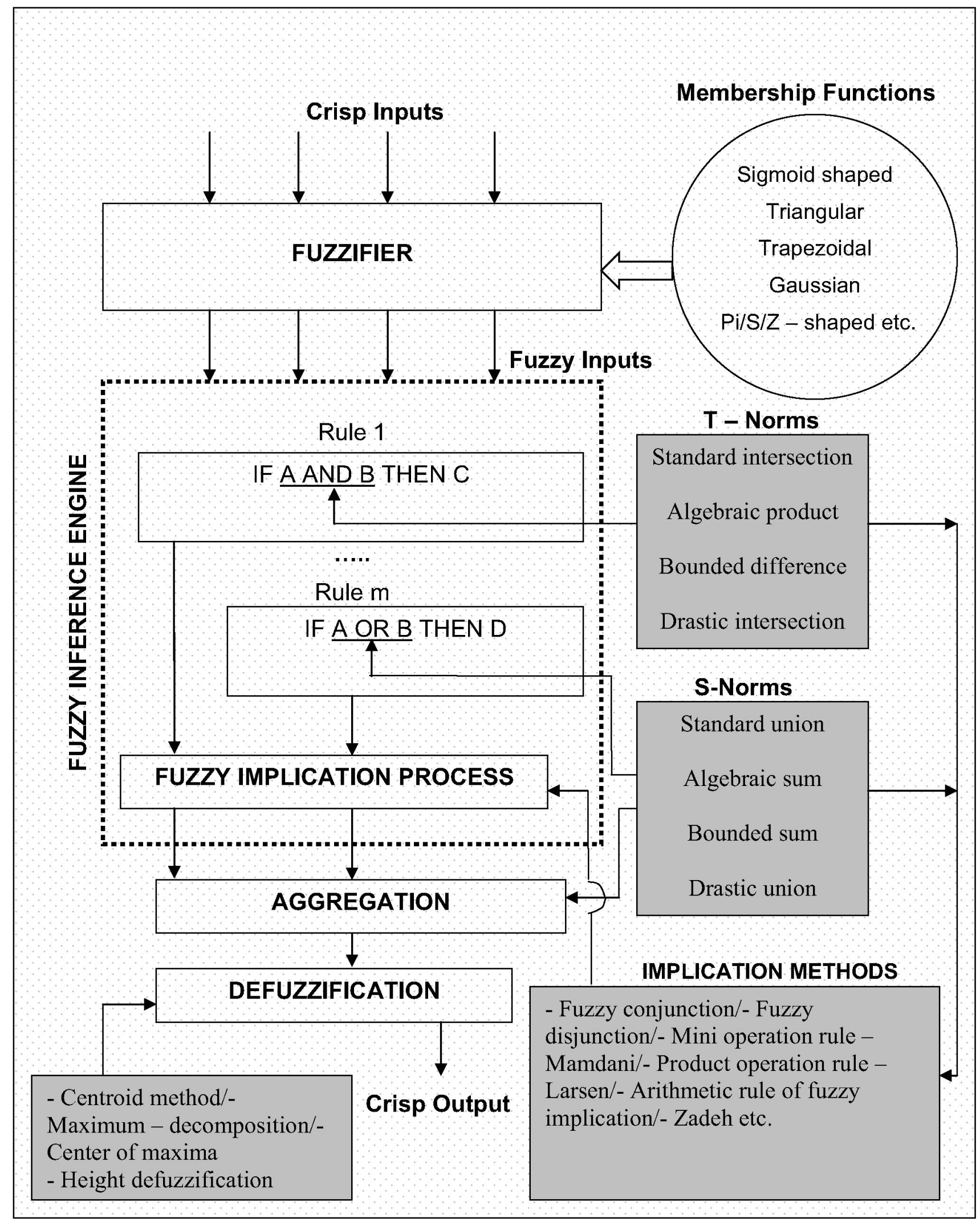

Fig. 5: Fuzzy Inference system and Macro level process 